\title{
The Ras/Raf/MEK/ERK signaling pathway and its role in the occurrence and development of HCC (Review)
}

\author{
LEI LI, GUO-DONG ZHAO, ZHE SHI, LI-LI QI, LI-YUAN ZHOU and ZE-XIAN FU \\ Department of General Surgery, Affiliated Hospital of Hebei University of Engineering, \\ Handan, Hebei 056029, P.R. China
}

Received February 15, 2016; Accepted July 13, 2016

DOI: $10.3892 / \mathrm{ol} .2016 .5110$

\begin{abstract}
Hepatocellular carcinoma (HCC) is the fifth most common tumor worldwide and has a very poor prognosis. Its occurrence has been on the increase in recent years. Surgical resection and liver transplantation are the primary methods of treatment for HCC patients, but can only be applied to $15 \%$ of patients. The median survival time of unresectable or metastasizing HCC patients is only a few months. Existing systemic treatment methods are not effective for advanced HCC patients and a new method of treatment is needed for these patients. It has been established that the HCC occurs in multiple stages, however, the pathogenesis at a molecular level is not clear and many key factors are yet to be determined. In the past 30 years, it has become evident that the Ras/Raf/MEK/extracellular signal-regulated kinase (ERK) signaling pathway plays a significant role in the occurrence and development of HCC. This review focused on the association between the Ras/Raf/MEK/ERK signaling pathway and HCC.
\end{abstract}

\section{Contents}

1. Introduction

2. Mechanism of Ras/Raf/MEK/ERK cascade reaction

3. The role of Ras/Raf/MEK/ERK pathway in tumor cells

4. Tumor treatment by inhibiting Ras/Raf/MEK/ERK pathways

5. The role of Ras/Raf/MEK/ERK pathway in liver cancer

\section{Introduction}

Hepatocellular carcinoma (HCC), is the fifth most common tumor worldwide and has a poor prognosis. Its occurrence has

Correspondence to: Dr Ze-Xian Fu, Department of General Surgery, Affiliated Hospital of Hebei University of Engineering, 81 Congtai Road, Handan, Hebei 056029, P.R. China

E-mail: fsyylilei@163.com

Key words: hepatocellular carcinoma, Ras, Raf, MEK, extracellular signal-regulated kinase been on the increase in recent years. The estimated incidence of new cases is approximately 500,000-1,000,000 annually, causing 600,000 mortalities globally per year (1).Surgical resection and liver transplantation are the primary methods of treatment for HCC patients, but can only be applied to $15 \%$ of patients $(2,3)$. Previous studies have demonstrated that the Ras/Raf/MEK/extracellular signal-regulated kinase (ERK) signaling pathway is important in the occurrence and development of HCC (3). This review focused on the association between the Ras/Raf/MEK/ERK signaling pathway and HCC.

\section{Mechanism of Ras/Raf/MEK/ERK cascade reaction}

Mitogen-activated protein kinase (MAPK) is a serine/threonine protein kinase that is widely present in eukaryotic cells. Previous studies found four different MAPKs: i) ERK; ii) c-Jun $\mathrm{N}$-terminal kinase (JNK); iii) ERK5; and iv) p38 MAPK (p38) (4). ERK cascade reaction can be activated by various stimuli, such as receptor tyrosine kinase (RTK) and G protein-coupled receptors. After activation it can regulate the proliferation, differentiation and apoptosis (5).

Ras/Raf/MEK/ERK cascade reaction is an important signaling pathway in MAPKs. Various stimuli can activate the corresponding cell surface receptors that, in turn, activates the signal transduction pathway and produce an appropriate biological response (6). The Ras/Raf/MEK/ERK cascade reaction is the key factor in integrating the signal transduction pathway. By transmitting the signals received by the receptor, Ras/Raf/MEK/ERK cascade reaction activates transcription factors and regulates gene expression. Briefly, the process involves: i) Ras recruits and activates the protein kinase Raf; ii) Raf serine/threonine protein kinase promotes MEK1/2 (MAPK/ERK kinase) dual-specificity protein kinase and the activation of ERK1/2; and iii) activated ERK1/2 phosphorylated several substrates and regulate different transcription factors, leading to different gene expression (7).

\section{The role of Ras/Raf/MEK/ERK pathway in tumor cells}

Various mechanisms can activate the Ras/Raf/MEK/ERK cascade in tumor cells: i) chromosome ectopic, such as BCR-ABL; ii) cytokine mutations, such as Flt-3, Fms and Kit; and iii) overexpression of wild or mutant receptors, such as epidermal growth factor receptor (EGFR). Ras/Raf/MEK/ERK 
cascade plays an important role in the apoptosis process by phosphorylating various apoptosis regulating factors, such as Bad, Bim, Mcl-1, caspase-9 and the controversial Bcl-2. Ras/Raf/MEK/ERK cascade plays a variety of roles in cell cycle regulation, apoptosis and cell differentiation (8). Increasing evidence regarding Ras/Raf/MEK/ERK cascade has focused on kinases, transcription factors, apoptosis regulatory factors and the caspase family (9).

Kinase proteins in the Ras/Raf/MEK/ERK pathway. Ras is a type of small GTP-binding protein. It is the upstream molecule in various pathways, such as MEK/ERK, PI3K/Akt, and RalEGF/Ral pathways (10). Ras has the Ha-Ras, N-Ras, Ki-Ras 4A and Ki-Ras 4B subtypes, of which Ki-Ras 4A and $\mathrm{Ki}-\mathrm{Ras} 4 \mathrm{~B}$ are derived from the same gene in different cutting positions (11). The effects of different subtypes in mediating different pathways are varied. Ki-Ras is stronger than Ha-Ras in MEK/ERK pathway (12), but Ha-Ras induction in PI3K/Akt pathway is stronger than that in MEK/ERK pathway. The mutation frequency of different Ras subtypes in human cancers is also different. The mutation frequency of Ki-Ras is higher in most type of cancer while the mutation frequency of N-Ras is higher only in some cancers. Ras in situ gene amplification and abnormal activation, leading to a sustained expression of Ras, was found in $30 \%$ of human cancers (13).

Raf family members (Raf-1, B-Raf, and A-Raf) are Ras effectors and upstream activators of the ERK pathway. Raf, a serine/threonine protein kinase, can phosphorylate proteins directly or promote protein phosphorylation via MEK/ERK activation downstream and regulate the apoptotic process. Previous studies focused on Ras/Raf/MEK/ERK pathway activation produced by Ras mutation and overlooked the Raf mutation. Recent studies have revealed that the B-Raf mutation rates are high in certain cancers, for example, the B-Raf mutation rate is $27-70 \%$ in melanoma, $36-53 \%$ in papillary thyroid carcinoma, $5-22 \%$ in colon carcinoma, and $30 \%$ in ovarian cancer (14-17). Mutation may occur in different positions in B-Raf protein; however the majority of common mutations occur at the residue number 600 , which replaces valine to glutamic acid (V600E) (14). V600E mutation accounts for $90 \%$ of Raf mutations in melanoma and thyroid cancer (9). $\mathrm{B}-\mathrm{Raf}$ is considered to be the most important kinase in the Ras/Raf/MEK/ERK cascade reactions (14). The mutated B-Raf can activate MEK and ERK downstream, and activate Raf-1 in wild-type and mutant-type B-Raf, thus further activating MEK and ERK (18).

MEK1 and MEK2 are related protein kinases involved in the Ras/Raf/MEK/ERK signal transduction cascade. This cascade participates in the regulation of a large variety of processes including apoptosis, cell cycle progression, cell migration, differentiation, metabolism, and proliferation. MEK1/2 are tyrosine and serine/threonine dual specificity protein kinases. MEK1 is activated by the phosphorylation of S218 and S222 in its activation segment as catalyzed by RAF kinases. The three members of the Raf family may promote MEK activation, but their biological effectiveness is dissimilar (B-Raf > Raf-1 >> A-Raf) (19). The mutant-type MEK activation eliminates the cytokine dependence of hematopoietic cells and leads NIH-3T3 to morphological changes (20).
ERK1/2 are members of the MAPK super family that can mediate cell proliferation and apoptosis. Thus ERK is a key member in the Ras/Raf/MEK/ERK signaling pathway mediated by Raf. ERK1/2 are two subtypes of highly conservative serine/threonine kinase, which can be activated by the dual specificity of kinase MEK1/2. The activated EPK can phosphorylate corresponding protein kinases in cytoplasm, cell membrane, and nucleus, thus extending and diversifying the cascade reaction of the signaling pathway. There are as many as 160 target molecules in the downstream of ERK1/2 (9). ERK1/2 can lead to the phosphorylation of different transcription factors, such as Ets-1, c-Jun, c-Myc and $\mathrm{NF}-\kappa \mathrm{B}$ (21). It can also activate transcription factor CREB through phosphorylation of Rsk (ribosomal S6 kinase) (8). With the exception of ERK, no other kinase was capable of activating various downstream substrates. Previous findings showed that even subtle changes in ERK activation can lead to its regulatory effects (22).

Ras/Raf/MEK/ERK pathway plays a role in drug resistance. The Ras/Raf/MEK/ERK cascade reaction was related to cell resistance to chemotherapy. By isolating the cells with adriamycin and paclitaxel resistance from the cytokine-dependent FL5.12 cells, McCubrey et al found that these cells had increased ERK expression and were highly sensitive to Raf/MEK inhibitors (9). Other studies on the MEK gene-deficient cells showed that the activation of MEK can increase the resistance of cells towards adriamycin 10-fold (9). On the other hand, the sensitivity of deficient cells to adriacin was improved. Zhang et al used HCC cells to show that the resistance to chemotherapy drug 5-FU was closely associated with the basic expression of p-ERK (7). In breast cancer, activated Raf was able to lead to doxorubicin and paclitaxel resistance by expressing drug-pump Mdr-1 and Bcl-2 anti-apoptotic proteins (9). These conclusions provide clues for further improvement of curative effects by blocking the Ras/Raf/MEK/ERK pathway to reduce drug resistance.

Raf/MEK/ERK pathway may interact with other pathways. The effect of Ras/Raf/MEK/ERK pathway can be enhanced or weakened by other signal transduction pathways. A study on cytokine dependence of FL5.12 cells revealed that cytokine dependence was not significantly decreased when Raf, MEK, PI3K, or Akt were activated independently (23). Nevertheless, cytokine dependence was significantly weakened when Raf/MEK and PI3K/Akt pathways were activated simultaneously by introducing activated Raf and Akt genes. This result indicated that Raf/MEK and PI3K/Akt signaling pathways were able to enhance cell proliferation by interacting with each other (23).

\section{Tumor treatment by inhibiting Ras/Raf/MEK/ERK pathways}

In recent years, Ras/Raf/MEK/ERK signal transduction pathways have become important targets in cancer treatment research. Inhibitors of Ras, Raf, MEK, ERK and other downstream molecules have been developed and partially used in clinical trials (24). Since MEK inhibitors are not ATP competitors, they have become of interest to cancer 
researchers. ERK, due to its ATP-biding pocket, which is similar to cycle-dependent proteins, was not inhibited. Thus, ERK inhibitors do not constitute the focus of attention for investigators.

The development of MEK inhibitor in preclinical application. From the results obtained from experiments on mice with colon cancer cells, it was found that as much as $80 \%$ of tumor growth was inhibited by MEK inhibitor PD184352 (also known as CI-1040) (25). It has been shown that mutant-type B-Raf cells had a higher sensitivity to MEK inhibitors (26). In the mutant-type B-Raf cells, inhibition of ERK activity by PD184352 effectively reduced cyclin D1 expression, while in normal B-Raf cells, inhibition of ERK activation by PD184352 failed to effectively reduce cyclin D1 expression. To reduce cyclin D1 expression, a higher dose of MEK inhibitors was required. Compared to the wild-type B-Raf cells, mutant-type B-Raf cells required a much smaller dose of MEK inhibitors to produce a $>50 \%$ cell proliferation inhibition rate $(26)$. The results obtained from the animal experiments have promoted the clinical trial of MEK inhibitors in cancer treatment. For example, in breast, colon, non-small cell lung and pancreatic cancer, PD184352 (CI-1040) reached phase II trials, where its antitumor efficacy and safety was assessed. However, PD184352 did not reveal effective antitumor properties in the abovementioned cancers, clinical trials had to be suspended (27). The failure of PD184352 may be explained by in vitro trails of a new MEK inhibitor, PD184161. Klein et al (28) carried out a study on the effects of PD184161 by inoculating hepatoma carcinoma cells into nude mice. The results of that study showed that PD184161 inhibited the tumor formation in nude mice but was not significantly effective in tumorigenic mice. When PD184161 was applied to cells and tumor cells for the first time, MEK activation was significantly reduced. However, in the long term, MEK expression was gradually restored to normal levels, indicating that the cells that survived the first round of drug therapy adapted to MEK inhibitors and rapidly lost their high sensitivity. Those results were confirmed by several experiments on tumor cells (26). In the $2005 \mathrm{ASCO}$ meeting, a more potent MEK inhibitor PD0325901 was introduced. PD0325901, which is now in phase II clinical trials, was found to exert a strong inhibitory effect on ERK activation (29).

Raf inhibitors are in the trial phase. Experiments on Raf inhibitor BAY43-9006 suggested that B-Raf was active in melanoma cells and reducing B-Raf expression had an inhibitory effect on the cell cycle process and improved the cell apoptotic rate (22). Thus, BAY43-9006 has also been employed in clinical trials.

Evidence suggests that tumor stem cells are present in many tumor cells (30) and although cancer stem cells accounted for only a small part of the tumors (0.1-2\%), they were able to independently express a high level of MEK/ERK. The embryonic stem cell proliferation did not require any ERK activation; by contrast, a high level of ERK expression affected the self replication of embryonic stem cells and promoted their differentiation (31). If characteristics of tumor stem cells were similar to those of embryonic stem cells, MEK inhibitors may not be able to remove the tumor but promote tumor recurrence.

\section{The role of Ras/Raf/MEK/ERK pathway in liver cancer}

Increased expression of Ras/Raf/MEK/ERK kinase in HCC tissue and HCC cells. The Ras/Raf/MEK/ERK pathway is important in the pathogenesis of HCC. Firstly, the Ras gene that acted as an upstream component of the signal transduction pathway was mutated in $30 \%$ of HCC cases (32). Secondly, Raf kinase was overexpressed in most HCC cases (33). Thirdly, the general overexpression of many upstream growth factors in HCC, such as EGF, vascular endothelial growth factor (VEGF), platelet-derived growth factor- $\beta$, (PDGF- $\beta)$ and transforming growth factor- $\alpha$ (TGF- $\alpha$ ) were combined with RTK and activated the Ras/Raf/MEK/ERK pathway (34).

In several human primary tumors and cell lines we observe active ERK. Active ERK has been reported in $36 \%$ of 102 primary tumor tissues and 138 tumor cell lines (35). Oka et al reported that ERK expression in tumor tissues was 1.5- to 32.9-fold higher than that in normal tissues (36). In breast cancer, ERK expression in tumor tissues was 5- to 10-fold higher than that in para-carcinoma tissues (37). Yoshida et al found that p-ERK expression in 53\% HCC tissues was higher than that in para-carcinoma tissues (38). Ito et al study revealed that ERK expression in HCC tissues was 1.1- to 3.1-fold higher than that in para-carcinoma tissues, and the ERK activation level in para-carcinoma tissues in metastatic HCC was lower than the average level of para-carcinoma tissues in the liver carcinomas, indicating that ERK level of para-carcinoma tissues in liver carcinoma was significantly higher than that in normal liver tissues (39). These levels were slightly lower than the level observed in renal carcinoma and breast cancer and were different from the para-carcinoma tissues in renal carcinoma and breast cancer. Para-carcinoma tissues in HCC were accompanied with regeneration and reconstruction resulting from chronic inflammations, such as chronic hepatitis and cirrhosis. For cases with nerve damages, the joint effects of regeneration and inflammation led to increased ERK activity of HCC para-carcinoma tissues (40).

Notably, to what extent ERK needed to be activated to accelerate cell proliferation and/or differentiation remained to be determined. Ito et al found by transferring insulin receptor substrate-1 (IRS-1) to NIH/3T3 cells, the IRS-1 expression level increased and ERK activity was amplified 2- to 3-fold compared to the control group (41). IRS-1 overexpression in HCC Hep3 cells is capable of increasing the activation level of ERK by a factor of $1.4 \mathrm{X}$ and reduce the serum requirements and cell apoptosis mediated by transfer growth factor (42). Consequently in HCC, even a low level of ERK activity was able to surpass the compensatory mechanism regulation on cell growth and transmit effective signals to downstream.

ERK phosphorylation activates a variety of target molecules to promote the development of liver cancer. Previous findings showed that ERK was mainly found in the HCC cell nucleus; thus, it was noteworthy to establish the existing relationship between ERK and the gene expression. It was established that ERK, after being activated, entered the nucleus (43) and activated some transcription factors via phosphorylation.ERK would induced c-Fos to combine with serum response factors through phosphorylation of tertiary complex factors/Ets-like 
kinase-1 (44). The kinase-defective mutated ERK inhibited the expression and transformation of c-Fos (45). ERK also activated c-Jun through phosphorylation (46). c-Fos and c-Jun jointly constituted the transcription activation factor-1 (ap-1), including c-Jun homodimer, c-Fos and c-Jun heterodimer (47). Transcription activating factor-1 is able to combine with the transcription activating factor- 1 binding site in the upstream promoter region and induce the transcription of multiple genes, including cell cycle protein D1 (cyclin D1) (48). Cyclin D1 is required for progression through the G1 phase of the cell cycle and is degraded as the cell enters the S phase (48). Cyclin D1 was found to be excessively expressed in HCC (49). In addition, cell cycle protein D1 is capable of inducing the instability and DNA amplification, resulting in the variation and transformation of cells (50).

Ito et al found that the expression of c-Fos protein in $44 \%$ of HCC cases was higher than that in para-carcinoma tissues, and the c-Fos expression level was positively correlated with ERK protein expression (39). Cyclin D1 was transcribed and activated through ERK. In the absence of ERK kinase activity, cyclin D1 expression was also inhibited (51). These results indicated that cyclin D1 expression may be mediated by c-Fos expression induced by ERK. Additionally, transcription factor-1 induced the transcription of matrix metalloproteinases (MMP). MMP in many malignant tumors either hydrolyzed components of the extracellular matrix or reconstructed extracellular matrix, thereby promoting invasion and metastasis (52). This mechanism may also exist in HCC and result in tumor invasion and metastasis.

Tumor characteristics markedly change when the liver's carcinoma diameter reach to approximately $2 \mathrm{~cm}$. It was shown that in the case of smaller than 2-cm liver tumors, only $10 \%$ grew by $>1.5$-fold in size, while $50 \%$ of larger liver tumors (with diameter over $2 \mathrm{~cm}$ ) grew by $>1.5$-fold in size (53). Ito et al showed that, an increase in tumor size led to an increase in the ERK expression level (39). Their results revealed that an increase in the ERK expression level in larger tumors $(>2 \mathrm{~cm})$ was significantly higher than that in smaller tumors $(<2 \mathrm{~cm})(39)$.

p-ERK is the phosphorylated form of ERK and is considered a key component downstream of the Ras/Raf/MEK/ERK pathway. Zhang et al (7) measured the basal expression of p-ERK in four HCC cell lines with different invasion abilities and found that the basal expression of p-ERK in SMMC-7721, MHCC97-L, MHCC97-H and HCCLM6 increased in turn, and the basal expression of p-ERK in invasive MHCC97-H and HCCLM6 was significantly higher than that in the non-invasive SMMC-7721. Those findings indicated that Ras/Raf/MEK/ERK pathway may be associated with the invasion and metastasis of HCC.

Hepatocyte growth factor (HGF) can promote cell growth and inhibit cell proliferation. Tsukada et al (54) studied the relationship between HGF's inhibitive property on HepG2 cell proliferation and Ras/Raf/MEK/ERK signal transduction pathway. Their results showed that HepG2 cell proliferation can be inhibited by MEK blocker PD98059, and the inhibition was concentration-dependent. When PD98059 concentration reached $10 \mu \mathrm{mol}$, the activation level induced by ERK was decreased and the cell proliferation inhibited by HGF was completely restored. However, when the PD98059 concentration was increased or decreased, such inhibition was not completely restored. These results showed that the promotion and inhibition of HepG2 cell proliferation required ERK regulation, and these opposite effects were determined by the activation level of ERK.

Inhibition of Ras/Raf/MEK/ERK pathway inhibitors in $H C C$. The role played by MEK inhibitors PD98059, U0126 and ERK1/2 antisense oligonucleotide in the proliferation, apoptosis, cell cycle and tumor occurrence in HCC Hep3B cells and HepG2 cells was studied by Wiesenauer et al (55). It was revealed that MEK inhibitors suppressed ERK1/2 phosphorylation while the total expression of ERK was not altered significantly. Those authors also showed that ERK1/2 antisense oligonucleotide inhibited ERK1/2 protein expression. The inhibitory effect of PD98059, U0126 and ERK1/2 antisense oligonucleotides on the proliferation of Hep3B and HepG2 cells was concentration-dependent. The two MEK inhibitors played an efficient role against cancer by obstructing cell cycle, improving the apoptotic rate and decreasing tumor incidence. U0126 inhibited ERK1/2 phosphorylation more effectively and the antitumor effect exerted was stronger than that of PD98059. HepG2 cells showed more sensitivity towards the inhibitory effects of ERK1/2 phosphorylation and the effect of drug treatment was more obvious in these cells. Hep3B cells were more sensitive to ERK1/2 downregulation mediated by ERK1/2 antisense oligonucleotides. Cell growth inhibition was more pronounced compared to HepG2 cells. These results indicated that blocking the Ras/Raf/MEK/ERK signaling pathway had multiple anticancer properties, and such effects were dependent on the sensitivity of cells and the different inhibition modes of drugs in the Ras/Raf/MEK/ERK pathway (55).

Association between hepatitis virus and Ras/Raf/MEK/ERK pathway. In developing countries, especially Asian countries, chronic HBV or HCV are the main sources of HCC (56). The relationship between viral infection and the Ras/Raf/MEK/ERK signaling pathway suggested that HBV $\mathrm{X}$ protein can induce ERK activation (57). Results showing that the hepatitis $\mathrm{C}$ virus core protein can combine with $\mathrm{H}$-ras and transform myofibroblastic tumors (58), indicated that viral infection may be involved in the regulation of signal transduction and may be associated with ERK activation in HCC.

Schmitz et al showed that, HCC patients with hepatitis C infection demonstrated higher levels of p-ERK1/2 compared to HCC patients with hepatitis B virus and those without viral infection (59). The results of Sato et al suggested that the hepatitis $\mathrm{C}$ virus core protein and envelope glycoprotein E2 can activate ERK pathway (60). Hepatitis C virus infection was a major cause of chronic hepatitis, liver cirrhosis and HCC. However, the exact mechanism of hepatitis $\mathrm{C}$ virus action in the occurrence of HCC remains to be determined.

In conclusion, Ras/Raf/MEK/ERK signal transduction pathway activation was important in the occurrence and development of HCC. In addition, the Ras/Raf/MEK/ERK pathway inhibitors may provide an effective option for the treatment of $\mathrm{HCC}$ in the near future. 


\section{References}

1. Guthle M and Dollinger MM: [Epidemiology and risk factors of hepatocellular carcinoma]. Radiologe 54: 654-659, 2014.

2. Llovet JM, Bruix J and Gores GJ: Surgical resection versus transplantation for early hepatocellular carcinoma: clues for the best strategy. Hepatology 31: 1019-1021, 2000.

3. Abou-Alfa GK: Current and novel therapeutics for hepatocellular carcinoma. In: American Society of Clinical Oncology Educational Book. Perry MC (ed). ASCO, Alexandria, VA, pp192-197, 2004.

4. Iyoda K, Sasaki Y, Horimoto M, Toyama T, Yakushijin T, Sakakibara M, Takehara T, Fujimoto J, Hori M, Wands JR and Hayashi N: Involvement of the p38 mitogen-activated protein kinase cascade in hepatocellular carcinoma. Cancer Am Cancer Soc 97: 3017-3026, 2003

5. Huynh H, Nguyen TT, Chow KH, Tan PH, Soo KC and Tran E: Over-expression of the mitogen-activated protein kinase (MAPK) kinase (MEK)-MAPK in hepatocellular carcinoma: its role in tumor progression and apoptosis. BMC Gastroenterol 3: 19, 2003.

6. Chung E and Kondo M: Role of Ras/Raf/MEK/ERK signaling in physiological hematopoiesis and leukemia development. Immunol Res 49: 248-268, 2011

7. Zhang Z, Zhou X, Shen H, Wang D and Wang Y: Phosphorylated ERK is a potential predictor of sensitivity to sorafenib when treating hepatocellular carcinoma: evidence from an in vitro study. BMC Med 7: 41, 2009.

8. Steelman LS, Pohnert SC, Shelton JG, Franklin RA, Bertrand FE and McCubrey JA: JAK/STAT, Raf/MEK/ERK, PI3K/Akt and BCR-ABL in cell cycle progression and leukemogenesis Leukemia 18: 189-218, 2004

9. McCubrey JA, Steelman LS, Chappell WH, Abrams SL, Wong EW, Chang F, Lehmann B, Terrian DM, Milella M, Tafuri A, et al: Roles of the Raf/MEK/ERK pathway in cell growth, malignant transformation and drug resistance. Biochim Biophys Acta 1773: 1263-1284, 2007.

10. Peyssonnaux C, Provot S, Felder-Schmittbuhl MP, Calothy G and Eychène A: Induction of postmitotic neuroretina cell proliferation by distinct Ras downstream signaling pathways. Mol Cell Biol 20: 7068-7079, 2000

11. Chappell WH, Steelman LS, Long JM, Kempf RC, Abrams SL, FranklinRA,Basecke J,StivalaF,Donia M,FagoneP,Malaponte G, Mazzarino MC, Nicoletti F, Libra M, Maksimovic-Ivanic D, Mijatovic S, Montalto G, Cervello M, Laidler P, Milella M, Tafuri A, Bonati A, Evangelisti C, Cocco L, Martelli AM and McCubrey JA: Ras/Raf/MEK/ERK and PI3K/PTEN/Akt/mTOR inhibitors: Rationale and importance to inhibiting these pathways in human health. Oncotarget 2: 135-164, 2011.

12. Yan J, Roy S, Apolloni A, Lane A and Hancock JF: Ras isoforms vary in their ability to activate Raf-1 and phosphoinositide 3-kinase. J Biol Chem 273: 24052-24056, 1998.

13. Stirewalt DL, Kopecky KJ, Meshinchi S, Appelbaum FR, Slovak ML, Willman CL and Radich JP: FLT3, RAS, and TP53 mutations in elderly patients with acute myeloid leukemia. Blood 97: 3589-3595, 2001.

14. Garnett MJ and Marais R: Guilty as charged: B-RAF is a human oncogene. Cancer Cell 6: 313-319, 2004.

15. Davies H, Bignell GR, Cox C, Stephens P, Edkins S, Clegg S, Teague J, Woffendin H, Garnett MJ, Bottomley W, et al: Mutations of the BRAF gene in human cancer. Nature 417: 949-954, 2002.

16. Libra M, Malaponte G, Navolanic PM, Gangemi P, Bevelacqua V, Proietti L, Bruni B, Stivala F, Mazzarino MC, Travali S, et al: Analysis of BRAF mutation in primary and metastatic melanoma. Cell Cycle 4: 1382-1384, 2005

17. Fransén K, Klintenäs M, Osterström A, Dimberg J, Monstein HJ and Söderkvist P: Mutation analysis of the BRAF, ARAF and $R A F-1$ genes in human colorectal adenocarcinomas. Carcinogenesis 25: 527-533, 2004.

18. Rushworth LK, Hindley AD, O'Neill E and Kolch W: Regulation and role of Raf-1/B-Raf heterodimerization. Mol Cell Biol 26: 2262-2272, 2006.

19. Alessi DR, Saito Y, Campbell DG, Cohen P, Sithanandam G, Rapp U, Ashworth A, Marshall CJ and Cowley S: Identification of the sites in MAP kinase kinase-1 phosphorylated by p74raf-1. EMBO J 13: 1610-1619, 1994.

20. Blalock WL, Pearce M, Steelman LS, Franklin RA, McCarthy SA Cherwinski H, McMahon M and McCubrey JA: A conditionallyactive form of MEK1 results in autocrine tranformation of human and mouse hematopoietic cells. Oncogene 19: 526-536, 2000.
21. Nakano H, Shindo M, Sakon S, Nishinaka S, Mihara M, Yagita H and Okumura K: Differential regulation of IkappaB kinase alpha and beta by two upstream kinases, NF-kappaB-inducing kinase and mitogen-activated protein kinase/ERK kinase kinase-1. Proc Natl Acad Sci USA 95: 3537-3542, 1998.

22. Chambard JC1, Lefloch R, Pouysségur J and Lenormand P: ERK implication in cell cycle regulation. Biochim Biophys Acta 1773: 1299-1310, 2007

23. Shelton JG, Chang F, Lee JT, Franklin RA, Steelman LS and McCubrey JA: B-raf and insulin synergistically prevent apoptosis and induce cell cycle progression in hematopoietic cells. Cell Cycle 3: 189-196, 2004.

24. Friday BB, Adjei AA: Advances in targeting the Ras/Raf/ MEK/Erk mitogen-activated protein kinase cascade with MEK inhibitors for cancer therapy. Clin Cancer Res 4: 342-346, 2008

25. Sebolt-Leopold JS, Dudley DT, Herrera R, Van Becelaere K, Wiland A, Gowan RC, Tecle H, Barrett SD, Bridges A, Przybranowski S, et al: Blockade of the MAP kinase pathway suppresses growth of colon tumors in vivo. Nat Med 5: 810-816, 1999.

26. Solit DB, Garraway LA, Pratilas CA, Sawai A, Getz G, Basso A, Ye Q, Lobo JM, She Y, Osman I, et al: BRAF mutation predicts sensitivity to MEK inhibition. Nature 439: $358-362,2006$

27. Rinehart J, Adjei AA, Lorusso PM, Waterhouse D, Hecht JR, Natale RB, Hamid O, Varterasian M, Asbury P, Kaldjian EP, et al: Multicenter phase II study of the oral MEK inhibitor, CI-1040, in patients with advanced non-small-cell lung, breast, colon, and pancreatic cancer. J Clin Oncol 22: 4456-4462, 2004.

28. Klein PJ, Schmidt CM, Wiesenauer CA, Choi JN, Gage EA, Yip-Schneider MT, Wiebke EA, Wang Y, Omer C and Sebolt-Leopold JS: The effects of a novel MEK inhibitor PD184161 on MEK-ERK signaling and growth in human liver cancer. Neoplasia 8: 1-8, 2006.

29. Leyton J, Smith G, Lees M, Perumal M, Nguyen QD, Aigbirhio FI, Golovko O, He Q, Workman P and Aboagye EO: Noninvasive imaging of cell proliferation following mitogenic extracellular kinase inhibition by PD0325901. Mol Cancer Ther 7: 3112-3121, 2008.

30. Jordan CT, Guzman ML and Noble M: Cancer stem cells. N Engl J Med 355: 1253-1261, 2006.

31. Burdon T, Smith A and Savatier P: Signalling, cell cycle and pluripotency in embryonic stem cells. Trends Cell Biol 12: 432-438, 2002

32. Downward J: Targeting RAS signalling pathways in cancer therapy. Nat Rev Cancer 3: 11-22, 2003.

33. Hwang YH, Choi JY, Kim S, Chung ES, Kim T, Koh SS, Lee B, Bae SH, Kim J and Park YM: Over-expression of $c$-raf-1 proto-oncogene in liver cirrhosis and hepatocellular carcinoma. Hepatol Res 29: 113-121, 2004

34. Roberts PJ and Der CJ: Targeting the Raf-MEK-ERK mitogen-activated protein kinase cascade for the treatment of cancer. Oncogene 26: 3291-3310, 2007.

35. Hoshino R, Chatani Y, Yamori T, Tsuruo T, Oka H, Yoshida O, Shimada Y, Ari-i S, Wada H, Fujimoto J, et al: Constitutive activation of the 41-/43-kDa mitogen-activated protein kinase signaling pathway in human tumors. Oncogene 18: 813-822, 1999.

36. Oka H, Chatani Y, Hoshino R, Ogawa O, Kakehi Y, Terachi T, Okada Y, Kawaichi M, Kohno M and Yoshida O: Constitutive activation of mitogen-activated protein (MAP) kinases in human renal cell carcinoma. Cancer Res 55: 4182-4187, 1995.

37. Sivaraman VS, Wang H, Nuovo GJ and Malbon CC: Hyperexpression of mitogen-activated protein kinase in human breast cancer. J Clin Invest 99: 1478-1483, 1997.

38. Yoshida T, Hisamoto T, Akiba J, Koga H, Nakamura K, Tokunaga Y, Hanada S, Kumemura H, Maeyama M, Harada M, et al: Spreds, inhibitors of the Ras/ERK signal transduction, are dysregulated in human hepatocellular carcinoma and linked to the malignant phenotype of tumors. Oncogene 25: 6056-6066, 2006.

39. Ito Y, Sasaki Y, Horimoto M, Wada S, Tanaka Y, Kasahara A, Ueki T, Hirano T, Yamamoto H, Fujimoto J, et al: Activation of mitogen-activated protein kinases/extracellular signal-regulated kinases in human hepatocellular carcinoma. Hepatology 27: 951-958, 1998.

40. Kitahara T, Kiryu S, Ohno K, Morita N, Kubo T and Kiyama H: Up-regulation of ERK (MAP kinase) and MEK (MAP kinase kinase) transcription after rat facial nerve transection. Neurosci Res 20: 275-280, 1994. 
41. Ito T, Sasaki Y and Wands JR: Overexpression of human insulin receptor substrate 1 induces cellular transformation with activation of mitogen-activated protein kinases. Mol Cell Biol 16: 943-951, 1996.

42. Tanaka S and Wands JR: Insulin receptor substrate 1 overexpression in human hepatocellular carcinoma cells prevents transforming growth factor betal-induced apoptosis. Cancer Res 56: 3391-3394, 1996.

43. Lenormand P, Sardet C, Pagès G, L'Allemain G, Brunet A and Pouysségur J: Growth factors induce nuclear translocation of MAP kinases (p42mapk and p44mapk) but not of their activator MAP kinase kinase (p45mapkk) in fibroblasts. J Cell Biol 122: 1079-1088, 1993.

44. Marais R, Wynne J and Treisman R: The SRF accessory protein Elk-1 contains a growth factor-regulated transcriptional activation domain. Cell 73: 381-393, 1993.

45. Kortenjann M, Thomae O and Shaw PE: Inhibition of $\mathrm{v}$-raf-dependent c-fos expression and transformation by a kinase-defective mutant of the mitogen-activated protein kinase Erk2. Mol Cell Biol 14: 4815-4824, 1994.

46. Pulverer BJ, Kyriakis JM, Avruch J, Nikolakaki E and Woodgett JR: Phosphorylation of c-jun mediated by MAP kinases. Nature 353: 670-674, 1991.

47. Chiu R, Boyle WJ, Meek J, Smeal T, Hunter T and Karin M: The c-Fos protein interacts with c-Jun/AP-1 to stimulate transcription of AP-1 responsive genes. Cell 54: 541-552, 1988.

48. Herber B, Truss M, Beato M and Müller R: Inducible regulatory elements in the human cyclin D1 promoter. Oncogene 9: 1295-1304, 1994.

49. Nishida N, Fukuda Y, Komeda T, Kita R, Sando T, Furukawa M, Amenomori M, Shibagaki I, Nakao K, Ikenaga M, et al: Amplification and overexpression of the cyclin D1 gene in aggressive human hepatocellular carcinoma. Cancer Res 54: 3107-3110, 1994.

50. Zhou P, Jiang W, Weghorst CM and Weinstein IB: Overexpression of cyclin D1 enhances gene amplification. Cancer Res 56: 36-39, 1996.

51. Lavoie JN, L'Allemain G, Brunet A, Müller R and Pouysségur J: Cyclin D1 expression is regulated positively by the p42/p44MAPK and negatively by the p38/HOGMAPK pathway. J Biol Chem 271: 20608-20616, 1996.
52. Noël A, Emonard H, Polette M, Birembaut P and Foidart JM: Role of matrix, fibroblasts and type IV collagenases in tumor progression and invasion. Pathol Res Pract 190: 934-941, 1994.

53. Ebara M, Ohto M, Shinagawa T, Sugiura N, Kimura K, Matsutani S, Morita M, Saisho H, Tsuchiya Y and Okuda K: Natural history of minute hepatocellular carcinoma smaller than three centimeters complicating cirrhosis. A study in 22 patients. Gastroenterology 90: 289-298, 1986.

54. Tsukada Y, Miyazawa K and Kitamura N: High intensity ERK signal mediates hepatocyte growth factor-induced proliferation inhibition of the human hepatocellular carcinoma cell line HepG2. J Biol Chem 276: 40968-40976, 2001.

55. Wiesenauer CA, Yip-Schneider MT, Wang Y and Schmidt CM: Multiple anticancer effects of blocking MEK-ERK signaling in hepatocellular carcinoma. J Am Coll Surg 198: 410-421, 2004.

56. Kudo M, Han KH, Kokudo N, Cheng AL, Choi BI, Furuse J, Izumi N, Park JW, Poon RT and Sakamoto M: Liver Cancer Working Group report. Jpn J Clin Oncol 40 (Suppl 1): i19-i27, 2010.

57. Doria M, Klein N, Lucito R and Schneider RJ: The hepatitis B virus HBx protein is a dual specificity cytoplasmic activator of Ras and nuclear activator of transcription factors. EMBO J 14: 4747-4757, 1995.

58. Ray RB, Lagging LM, Meyer K and Ray R: Hepatitis C virus core protein cooperates with ras and transforms primary rat embryo fibroblasts to tumorigenic phenotype. J Virol 70: 4438-4443, 1996.

59. Schmitz KJ, Wohlschlaeger J, Lang H, Sotiropoulos GC, Malago M, Steveling K, Reis H, Cicinnati VR, Schmid KW and Baba HA: Activation of the ERK and AKT signalling pathway predicts poor prognosis in hepatocellular carcinoma and ERK activation in cancer tissue is associated with hepatitis $\mathrm{C}$ virus infection. J Hepatol 48: 83-90, 2008.

60. Sato Y, Kato J, Takimoto R, Takada K, Kawano Y, Miyanishi K, Kobune M, Sato Y, Takayama T, Matunaga T, et al: Hepatitis C virus core protein promotes proliferation of human hepatoma cells through enhancement of transforming growth factor alpha expression via activation of nuclear factor-kappaB. Gut 55: 1801-1808, 2006. 